\title{
The Effect of Anterior Corneal Astigmatism Orientation on Toric Intraocular Lens Outcomes
}

\author{
Eugene A. Lowry ${ }^{1 *}$, Jonathan $\mathrm{Li}^{1 * \#, ~ S u n d e e p ~ K . ~ K a s i ², ~ S e t h ~ B l u m b e r g ~}{ }^{3}$, Priya Morjaria ${ }^{4}$, \\ Steven Schallhorn ${ }^{1}$, Ayman Naseri ${ }^{1,5}$
}

\footnotetext{
${ }^{1}$ Department of Ophthalmology, University of California San Francisco, San Francisco, CA, USA

${ }^{2}$ Retina Service of Wills Eye Hospital, Mid Atlantic Retina, Philadelphia, PA, USA

${ }^{3}$ Francis I. Proctor Foundation, University of California San Francisco, San Francisco, CA, USA

${ }^{4}$ Department of Clinical Research, London School of Hygiene and Tropical Medicine, London, UK

${ }^{5}$ Department of Ophthalmology, San Francisco VA Medical Center, San Francisco, CA, USA

Email: jonathan.li@ucsf.edu
}

How to cite this paper: Lowry, E.A., Li, J., Kasi, S.K., Blumberg, S., Morjaria, P., Schallhorn, S. and Naseri, A. (2019) The Effect of Anterior Corneal Astigmatism Orientation on Toric Intraocular Lens Outcomes. Open Journal of Ophthalmolo$g y, 9,84-93$.

https://doi.org/10.4236/ojoph.2019.92010

Received: March 5, 2019

Accepted: May 6, 2019

Published: May 9, 2019

Copyright $\odot 2019$ by author(s) and Scientific Research Publishing Inc. This work is licensed under the Creative Commons Attribution International License (CC BY 4.0).

http://creativecommons.org/licenses/by/4.0/

\begin{abstract}
Background: It is unclear whether post-operative errors after toric intraocular lens implantation would be more amenable to pre-operative correction with a fixed adjustment or a correction ratio that scales with the magnitude of pre-operative astigmatism. Purpose: To investigate the effect of pre-operative anterior corneal astigmatism orientation on outcomes of toric intraocular lens implantation in a large population. Methods: A retrospective cohort study of 625 patients undergoing refractive lens exchange through a superior clear corneal incision with Oculentis M-Plus toric intraocular lens implantation at an Optical Express, Inc. located in the United Kingdom and Ireland. Patients were stratified by axis of astigmatism on automated keratometry as with-the-rule, against-the-rule, or oblique. Analysis of visual acuity and refractive outcomes was performed using American National Standards Institute (ANSI) guidelines on astigmatic corrections with non-vector as well as vector analyses. Analysis was limited to one eye per patient. Results: Patients who had with-the-rule (WTR) astigmatism, compared with oblique and against-the-rule (ATR), had higher vector magnitudes of surgically induced refractive correction $(2.89 \mathrm{D}, 2.55 \mathrm{D}, 2.42 \mathrm{D} ; \mathrm{p}<0.001)$, and larger correction ratios $(1.18,1.02,1.07 ; \mathrm{p}<0.001)$. This created a change of astigmatic axis in the with-the-rule cohort from WTR pre-operative astigmatism to an average ATR post-operative astigmatism. The overcorrection in the WTR cohort averaged $0.30 \mathrm{D}$. We found that the absolute dioptric overcorrection among
\end{abstract}

${ }^{\star}$ First co-authors. 
WTR patients did not differ significantly with magnitude of corneal astigmatism, but the correction ratio was lower among those with higher baseline cylindrical errors ( $\mathrm{p}=0.93$ and 0.0002 , respectively). Conclusions: Refractive lens exchange surgery using toric intraocular lenses overcorrected patients who had with-the-rule astigmatism. Degree of overcorrection did not vary with severity of pre-operative astigmatism. Incorporation of axis of astigmatism in lens selection and reduction of astigmatic correction among with-the-rule patients by an absolute value of $0.25 \mathrm{D}-0.35 \mathrm{D}$, rather than proportional adjustments, may reduce cylindrical over-correction.

\section{Keywords}

Toric, Lens, Astigmatism, Cataract Surgery, Refractive Lens Exchange

\section{Introduction}

Toric intraocular lenses are utilized in cataract and refractive lens exchange surgery for patients with corneal astigmatism to reduce spectacle dependence. The lens has a spherocylindrical correction and can be used to correct large powers of corneal cylinder [1] though residual astigmatism in the 0.7 to $1 \mathrm{D}$ range remains common [1] [2] [3] [4]. Traditional pre-operative measurements such as placido disc-based corneal topography and automated keratometry measure only the anterior corneal surface [5]. These measures differ significantly from Scheimpflug-based imaging that measures anterior and posterior corneal surface [6]. Differences between anterior and total corneal astigmatism may be as much as $0.5 \mathrm{D}$ or 10 -degrees in $28 \%$ of eyes, with most eyes having posterior corneal astigmatism steeper in the vertical direction [7] [8].

This discrepancy has led to investigations into whether orientation of keratometric astigmatism may affect the extent of cylindrical correction. Earlier studies found no difference in magnitude of residual cylinder or uncorrected distance visual acuity after toric lens implantation among eyes having WTR or ATR astigmatism on the basis of axis of astigmatism [9] [10]. Subsequent studies have shown a tendency for toric lens placement guided by automated keratometry to overcorrect WTR and undercorrect ATR patients [11] [12] [13]. However, these studies have included smaller cohorts of patients ranging from $35-143$ eyes and have variably reported the errors as average dioptric overcorrection [11] [12] or as a correction ratio that is proportional to pre-operative cylinder [13], making it unclear whether a fixed or proportional adjustment may be optimal. Given uncertainty of whether corrections should be a fixed pre-operative adjustment based solely on orientation of astigmatism or also scale with magnitude of pre-operative corneal cylinder [13], it is important to determine a more specific pre-operative adjustment recommendation.

In this study, we investigate differences in degree of astigmatic correction, residual astigmatism, and any correlation between baseline astigmatism and degree of overcorrection among a large group of patients who have toric lens im- 
plantation. In this larger population, we analyze whether any post-operative errors would be more amenable to pre-operative correction with a fixed adjustment or correction ratio that scales with the magnitude of pre-operative astigmatism.

\section{Patients and Methods}

This study was approved by the Committee on Human Research at the University of California, San Francisco and the London School of Hygiene and Tropical Medicine and adhered to the tenets of the Declaration of Helsinki.

The study group included all adult patients 70 years of age or younger who underwent primary refractive lens exchange with implantation of an Oculentis M-Plus Toric intraocular lens (Oculentis; Eerbeek, Netherlands) at Optical Express, Inc. in their United Kingdom and Ireland centers from 2010 to 2013 with at least $1.25 \mathrm{D}$ of pre-operative anterior corneal astigmatism. Patients were excluded if they had simultaneous additional intraocular surgeries, did not have follow-up at the final three-month outcome including manifest refraction and best-corrected visual acuity (BCVA), did not have a baseline BCVA and keratometry readings recorded in the electronic medical record, had a co-existing ophthalmic disease reducing potential visual acuity, or had greater than 3 diopters of surgically induced refractive change of the corneal surface on post-op month three keratometry, which was felt to be a marker of either data entry error or surgical complication. Patients older than 70 were excluded to reduce variation in lens density of these refractive surgeries, limiting to a population with softer lenses and more consistent surgical course. Manifest refraction was performed using a resolution-based technique in which the endpoint is the least amount of minus sphere that results in the best visual acuity ("push plus").

The following data were routinely recorded for all patients undergoing refractive lens exchange at Optical Express, Inc., and were gathered and analyzed for this study: patient sex and age, pre- and post-operative visual acuity, manifest refraction, pre-operative automated keratometry (performed with IOL Master in all cases). Post-op month three automated keratometry was also collected on the majority of patients. Patients were classified as having WTR, ATR, or oblique based on axis of astigmatism measured by automated keratometry in positive cylinder notation (WTR axis 60 - 120 degrees, ATR 0 - 30 or 150 - 180 degrees, oblique 30 - 60 degrees or 120 - 150 degrees).

\subsection{Operative Technique}

Refractive lens exchange with Oculentis M-Plus toric lens implantation was performed using a $2.6 \mathrm{~mm}$ clear corneal incision located at 12 o'clock for both left and right eyes by a total of six different surgeons at four Optical Express centers. The native lens was removed through a superior clear corneal incision for the main wound and phaco-emulsification with either a divide and conquer or chopping technique. The M-Plus toric lens is a one-piece spherocylindrical toric 
acrylic plate haptic with A-constant 118.2 that is individually customized in advance for each patient with refractive correction, including cylinder, customizable to $0.01 \mathrm{D}$ increments. The lens also includes an inferior $+3.00 \mathrm{D}$ near add embedded on the posterior surface. Intraocular lens power was selected using the online Oculentis toric IOL calculator (available at www.lentistoric.com) with each surgeon entering a unique value for surgically induced astigmatism ranging from 0.2 - 0.3D. A final target refraction of plano was assumed to be the goal of all surgeons given the lens allowed continuous dioptric and cylindrical correction and already included near add.

\subsection{Statistical Analysis}

Statistical analysis was performed using standard analyses of astigmatic correction recommended for laser systems that reshape the cornea adapted to intraocular lens implantation and included both non-vector and vector analyses [14]. For non-vector analysis, uncorrected visual acuity was compared at three months after surgery with Kruskal-Wallis testing. The proportion of patients achieving half to one diopter of mean-spherical and cylindrical goals and proportion achieving 20/40 or 20/20 or better vision were compared with Fisher's exact testing. Vector analysis of astigmatic correction is described in detail elsewhere [14]. In brief, initial refractive errors and surgical targets were based on pre-operative automated keratometry readings, while final refractive outcomes were based on post-op month three manifest refraction converted to the corneal plane assuming a $12 \mathrm{~mm}$ vertex distance for manifest refraction. The cylinder of all left eyes was flipped on the vertical axis to prevent cancellation when averaging right and left eyes and axis angles were doubled to convert to vector angles. We then determined the astigmatic intended refractive correction (IRC), surgically induced refractive correction (SIRC), error vector (EV; difference between IRC and SIRC), correction ratio (|SIRC|/|IRC|), error ratio $(|\mathrm{EV}| /|\operatorname{IRC}|)$, and treatment error vector (TEV, vector with length of magnitude of error and angle showing direction of error). We also correlate the pre-operative cylinder with both absolute diopters of overcorrection (defined as SIRC minus IRC) and correction ratio. We compare pre-operative with post-op month three corneal astigmatism magnitudes to determine surgically induced corneal astigmatism. Significance testing was performed with one-way ANOVA for log-MAR data and Kruskal-Wallis for other continuous variables. Confidence intervals were generated. When results were found to be significant below a $\mathrm{p}=0.05$ value in multi-way comparison, multiple pairwise comparisons were performed. These pairwise comparisons were adjusted for the three pairwise possibilities with a Holm-Bonferroni adjustment of multiple hypothesis testing. The magnitude of the intended astigmatic refractive correction for all patients was equal to the cylinder, as the target goal for residual astigmatism was zero. The metrics for refractive change were based on total astigmatic change of the eye, measuring the difference between pre-operative refraction and post-operative outcomes. Thus, these outcome measures included both toric correction and change in corneal 
astigmatism from incision incorporated into the definitions. For example, surgically induced astigmatism was the difference from pre- to post-operative astigmatism (the sum of changes from toric lens placement plus astigmatism change from corneal incision).

\section{Results}

1076 eyes of 714 patients underwent refractive lens exchange with placement of toric intraocular lens from 2010 to 2013. 153 eyes of 89 patients were excluded; with the majority of eyes (91/153) being excluded for no post-op month three vision or refraction and 22 excluded for age greater than 70 . This left 923 eyes of 625 patients, of which one eye was randomly selected for each patient, leaving 625 eyes of 625 patients for analysis. The large majority of these patients had with-the-rule astigmatism. There was no significant difference in baseline characteristics of age, sex, or magnitude of anterior corneal astigmatism measured by automated keratometry between the three groups (Table 1). There was a significant difference in eye laterality and baseline best corrected visual acuity between groups ( $p=0.03$ and 0.02 , respectively, Fisher's Exact Test). The majority of patients in all groups had good post-operative visual outcomes with an overall population mean uncorrected visual acuity of logMar 0.09 (approximately Snellen 20/25), mean best-corrected visual acuity of logMar -0.0005 (approximately Snellen $20 / 20$ ) and $94 \%$ of patients achieving uncorrected acuity of $20 / 40$ or better at three months (Figure 1).

Non-vector refractive analysis was performed. There was no significant difference in final uncorrected visual acuity between patients having WTR, ATR or oblique with respective $\log$ Mar acuities of $0.093,0.065$ and $0.133(p=0.07$, one-way ANOVA). Average magnitude of residual cylinder at the corneal plane on post-op month three manifest refraction was $0.69 \mathrm{D}, 0.62 \mathrm{D}$ and $0.88 \mathrm{D}$ in the WTR, ATR and oblique groups with no significant difference $(\mathrm{p}=0.05$, Kruskal-Wallis). There was no significant difference among cohorts in the proportion of patients within a half to one diopter of mean-spherical and cylindrical

Table 1. Baseline characteristics by type of astigmatism.

\begin{tabular}{ccccc}
\hline & With-the-rule & Against-the-rule & Oblique & P-value \\
\hline Number of Patients & 525 & 61 & 39 & NA \\
Right Eyes $(\mathrm{N}, \%)$ & $275(52 \%)$ & $31(51 \%)$ & $12(31 \%)$ & 0.03 \\
Age $( \pm$ SD) & $53.5 \pm 7.8$ & $53.6 \pm 9.1$ & $53.9 \pm 8.9$ & 0.93 \\
Sex $(\mathrm{N}, \%$ male $)$ & $219(42 \%)$ & $32(52 \%)$ & $19(49 \%)$ & 0.11 \\
$\begin{array}{c}\text { Pre-Operative BCVA } \\
\text { in LogMar }( \pm \text { SD) }\end{array}$ & $0.01 \pm 0.11$ & $-0.003 \pm 0.10$ & $0.05 \pm 0.11$ & 0.02 \\
$\begin{array}{c}\text { Magnitude of } \\
\text { Pre-Operative }\end{array}$ & & & & \\
$\begin{array}{c}\text { Corneal Astigmatism } \\
( \pm \text { SD) }\end{array}$ & $2.49 \pm 1.03$ & $2.29 \pm 1.34$ & $2.51 \pm 0.90$ & 0.76 \\
\hline
\end{tabular}

${ }^{a}$ Astigmatism grouping based on pre-operative automated keratometry. SD: Standard deviation. BCVA: best-corrected visual acuity. 


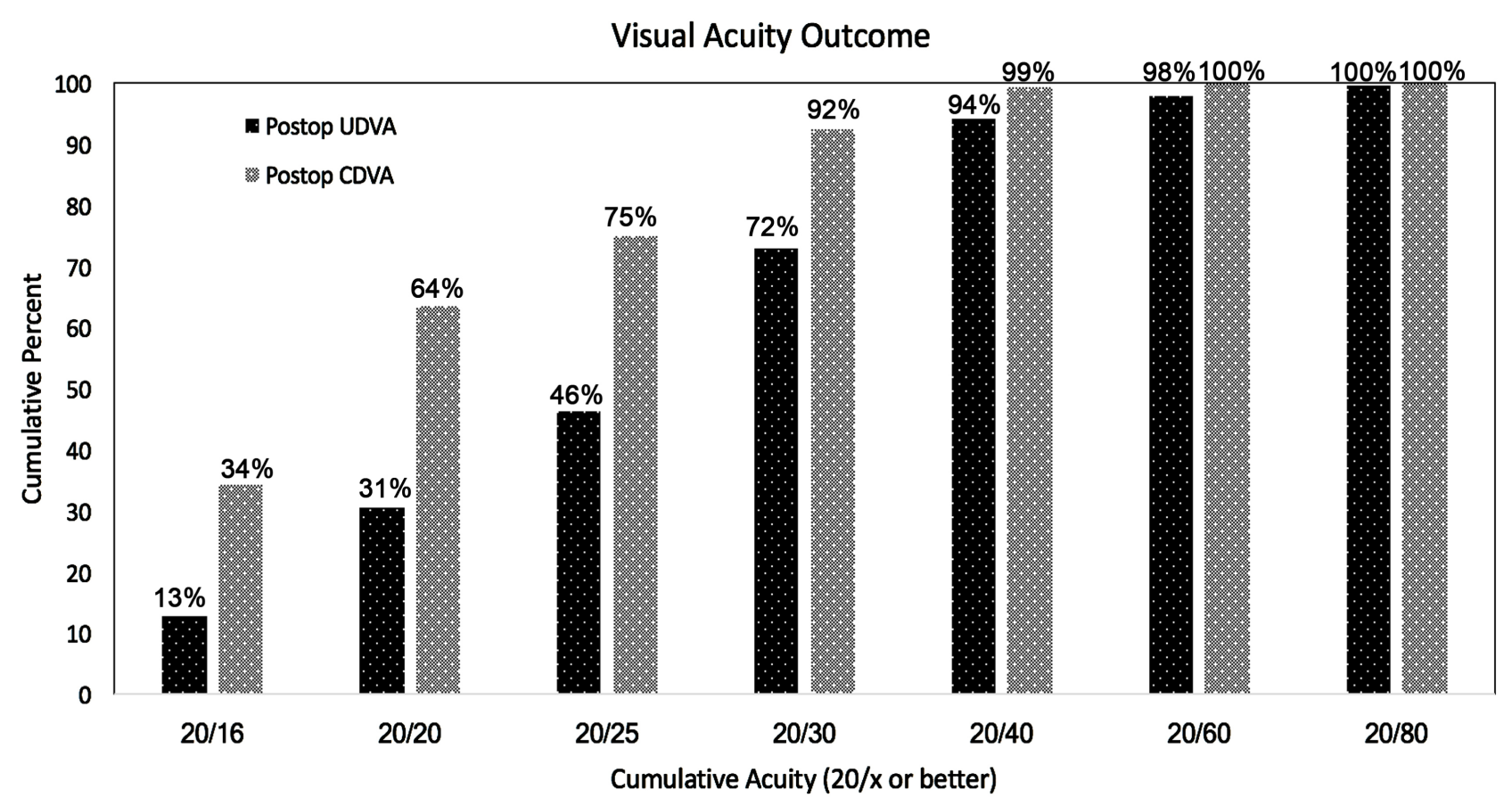

Figure 1. Post-operative visual outcomes. UDVA: Uncorrected distance visual acuity, CDVA: corrected distance visual acuity.

goals at post-op month three manifest or proportion of patients achieving 20/20 or better vision. In pairwise comparison at the 20/40 level, WTR had higher proportions of patients than the oblique cohort of achieving 20/40 or better ( $p=$ $0.04)$, though when corrected for multiple comparisons these pairwise differences no longer achieved significance $(\mathrm{p}=0.07)$ (Table 2).

Vector analysis of astigmatic refractive error was performed. Magnitude of intended refractive corrections were similar among all groups. After surgical correction, the WTR cohort was over-treated with a treatment error vector of $0.30 \mathrm{D} \times 89.9^{\circ}(95 \% \mathrm{CI}, 0.23 \mathrm{D}$ to $0.37 \mathrm{D})$ leaving residual ATR astigmatism with a mean error vector of $0.34 \mathrm{D} \times 7.1^{\circ}$. In contrast, the ATR cohort was undertreated with a mean treatment error vector of $0.12 \mathrm{D} \times 8.5^{\circ}$ (95\% CI, $0.02 \mathrm{D}$ to $0.21 \mathrm{D}$ ) leaving a mean error vector of $0.12 \mathrm{D} \times 36.7^{\circ}$. Averages of vector magnitude values tended to be larger than average vectors as different orientations did not partially cancel out (Table 3 ). While there was no difference in magnitude of intended refractive correction between groups $(\mathrm{p}=0.76)$, there was a significant difference in magnitude of surgically induced refractive correction with larger corrections in patients having WTR astigmatism compared with ATR and oblique ( $\mathrm{p}<0.001$, Kruskal-Wallis). This corresponded with a significantly greater correction ratio for patients having WTR astigmatism ( $\mathrm{p}<0.001$, Kruskal-Wallis), consistent with overcorrection of astigmatic errors (Table 3 ). We found that the absolute dioptric overcorrection among WTR patients did not differ significantly with magnitude of corneal astigmatism, while the correction ratio was lower among those with higher baseline cylindrical errors ( $p=0.93$ and 0.0002 , respectively). 
Table 2. Analysis of surgical outcomes by astigmatism class.

\begin{tabular}{ccccc}
\hline & With-the-rule & Against-the-rule & Oblique & P-value \\
\hline $\begin{array}{c} \pm 0.50 \mathrm{D} \text { MSE of goal } \\
(\mathrm{N}, \%)\end{array}$ & $369(70 \%)$ & $47(77 \%)$ & $29(74 \%)$ & 0.52 \\
$\begin{array}{c} \pm 1.0 \mathrm{D} \text { MSE of goal } \\
(\mathrm{N}, \%)\end{array}$ & $478(91 \%)$ & $55(90 \%)$ & $35(90 \%)$ & 0.88 \\
$\begin{array}{c}\text { Magnitude Residual } \\
\text { Cylinder }( \pm \text { SD) }\end{array}$ & $0.69 \pm 0.57$ & $0.62 \pm 0.53$ & $0.88 \pm 0.58$ & 0.05 \\
$\pm 0.5 \mathrm{D}$ of zero cylinder & $276(52 \%)$ & $35(57 \%)$ & $13(33 \%)$ & 0.05 \\
$\pm 1.0 \mathrm{D}$ of zero cylinder & $430(82 \%)$ & $51(84 \%)$ & $27(69 \%)$ & 0.14 \\
$\begin{array}{c}\text { Mean UCVA (logMAR) } \\
\text { UCVA 20/40 or better } \\
(\mathrm{N}, \%)\end{array}$ & $0.093 \pm 0.15$ & $0.065 \pm 0.14$ & $0.133 \pm 0.16$ & 0.07 \\
$\begin{array}{c}\text { UCVA } 20 / 20 \text { or better } \\
(\mathrm{N}, \%)\end{array}$ & $53(10 \%)$ & $59(97 \%)$ & $33(85 \%)$ & $0.04^{\mathrm{a}}$ \\
& & & $3(8 \%)$ & 0.88 \\
\hline
\end{tabular}

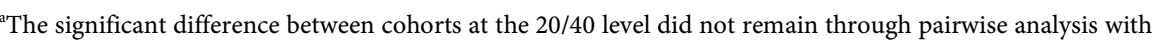
multiple hypothesis adjustment. D: Diopters. UCVA: Uncorrected visual acuity. MSE: mean spherical equivalent.

Table 3. Vector analysis of surgical outcomes by astigmatism class.

\begin{tabular}{ccccc}
\hline & With-the-rule & Against-the-rule & Oblique & P-value \\
\hline IRC Magnitude $( \pm \mathrm{SD})$ & $2.49 \pm 1.03$ & $2.29 \pm 1.33$ & $2.51 \pm 0.90$ & 0.76 \\
SIRC Magnitude $( \pm \mathrm{SD})$ & $2.89 \pm 1.13^{\mathrm{a}}$ & $2.42 \pm 1.27^{\mathrm{a}}$ & $2.55 \pm 1.01$ & $<0.001$ \\
Magnitude EV $( \pm \mathrm{SD})$ & $0.69 \pm 0.57$ & $0.62 \pm 0.52$ & $0.88 \pm 0.57$ & 0.06 \\
Correction Ratio $( \pm \mathrm{SD})$ & $1.18 \pm 0.33^{\mathrm{a}, \mathrm{b}}$ & $1.07 \pm 0.78^{\mathrm{a}}$ & $1.02 \pm 0.25^{\mathrm{b}}$ & $<0.001$ \\
Error Ratio $( \pm \mathrm{SD})$ & $0.31 \pm 0.34$ & $0.38 \pm 0.78$ & $0.39 \pm 0.29$ & 0.14 \\
\hline
\end{tabular}

${ }^{\text {aS }}$ Significant pairwise difference between with-the-rule and against-the-rule cohort with $\mathrm{p}$-value $<0.001$ after adjustments. ${ }^{b}$ Significant pairwise difference between with-the-rule and oblique cohort with $\mathrm{p}$-value $<0.001$ after adjustments. IRC: Intended refractive correction. SIRC: Surgically induced refractive correction. EV: Error Vector. Correction ratio is the ratio of magnitudes of SIRC divided by IRC. Error ratio is the magnitude of EV divided by IRC.

\section{Discussion}

This study investigated visual and refractive outcomes three months after refractive lens exchange with toric lenses among patients having with-the-rule, against-the-rule and oblique astigmatism. We found that patients having WTR astigmatism were significantly overcorrected compared to patients having ATR and oblique astigmatism. The mean vector error for residual astigmatism in the WTR cohort converted from a WTR axis at $91.4^{\circ}$ to ATR astigmatism at $7.1^{\circ}$. This change in axis was due to overtreatment in the WTR axis with a mean treatment error vector of $0.30 \mathrm{D}$ at $89.9^{\circ}$. The average correction ratio for eyes having WTR astigmatism (1.18) was significantly greater than ATR (1.07) and oblique (1.02) astigmatism $(\mathrm{p}<0.001)$. We found that absolute diopters of overcorrection did not vary significantly with pre-operative cylinder, while correction ratio did. 
The finding that absolute diopters of overcorrection was not found to significantly correlate with magnitude of pre-operative astigmatism was a novel finding of our study. Further, the magnitude of residual astigmatism seen here is similar to that of prior studies [11] [12], though the correction ratio is much closer to 1.0 than reported elsewhere [13] lending further support to the preferential treatment of constant rather than scalar pre-operative adjustments of astigmatic targets. Based on the confidence interval of the mean treatment error vector for WTR patients, we favor a fixed adjustment of approximately 0.25 $0.35 \mathrm{D}$ to refractive correction rather than an adjustment that scales with corneal power.

There may be several reasons for this overcorrection of WTR patients. The surgical technique in this study uses a superior clear corneal incision at 12 o'clock, a location associated with greater surgically induced astigmatism that may reduce some of the with-the-rule astigmatism (or increase against-the-rule astigmatism) [15]. However, the surgeons in this study estimated an average change in corneal astigmatism of $0.2-0.3 \mathrm{D}$ and we found an actual average change in the center of this range at $0.26 \mathrm{D}$ among the cohort of WTR who underwent repeated keratometry at post-operative month three. Given the lens selection was adjusted pre-operatively for this expected astigmatic change of the corneal incision, we believe the ongoing overcorrection of patients having WTR astigmatism is not explained by incision location. Measurement variations from any source including the automated keratometry machine and patient factors such as tear film may lead to inaccurate refractive outcomes, though these would be more likely to lead to random errors that would average out in a larger population rather than an average overcorrection of astigmatism. The measurements for IOL selections were based on keratometric measurements of the anterior corneal curvature with a correction for surgically induced astigmatism and do not specifically incorporate posterior corneal astigmatism. While we do not have data on total corneal astigmatism in our cohort, other studies have shown that incorporation of posterior measurements may lead to improved refractive outcomes [11] [12] [16].

The study has several limitations. The study was retrospective with the corresponding limitations, and prospective studies are merited. We assume that the target for residual astigmatism was zero given the M-Plus toric lens is continuously customizable on power and axis, though if residual cylinder was targeted, this would alter calculations of intended refractive correction. The population studied here was that of a refractive practice and generally younger with a high prevalence of WTR astigmatism. Orientation of astigmatism has been shown to become progressively more ATR with aging [17]. However, this would likely further exacerbate the study findings of induced post-operative ATR astigmatism in patients with initial WTR astigmatism, as their WTR astigmatism may tend to worsen over time. The primary indication for the procedure was generally refractive with an average pre-operative BCVA better than 20/25 in all groups studied. While this may limit generalizability to cataract extractions, the 
techniques and biometric considerations described here are otherwise the same as those used in cataract surgery. Finally, only anterior corneal curvatures were measured with automated keratometry, and we cannot definitively determine whether inclusion of total corneal astigmatism including posterior astigmatism would have led to better final refractive outcomes.

\section{Conclusion}

In conclusion, we found that there is a significant overcorrection of patients who have WTR astigmatism in refractive lens exchange with toric lens implantation using anterior corneal keratometric measurements from automated keratometry. Pre-operative consideration of axis of astigmatism and reductions in WTR correction by a fixed value of $0.25-0.35 \mathrm{D}$ rather than a correction ratio based on magnitude of pre-operative cylinder may further reduce residual refractive error and improve outcomes in surgeries using toric lenses.

\section{Conflicts of Interest}

The authors declare no conflicts of interest regarding the publication of this paper.

\section{References}

[1] Visser, N., Ruiz-Mesa, R., Pastor, F., Bauer, N.J., Nuijts, R.M. and Montés-Micó, R. (2011) Cataract Surgery with Toric Intraocular Lens Implantation in Patients with High Corneal Astigmatism. Journal of Cataract \& Refractive Surgery, 37, 1403-1410. https://doi.org/10.1016/j.jcrs.2011.03.034

[2] Ruhswurm, I., Scholz, U., Zehetmayer, M., Hanselmayer, G., Vass, C. and Skorpik, C. (2000) Astigmatism Correction with a Foldable Toric Intraocular Lens in Cataract Patients. Journal of Cataract \& Refractive Surgery, 26, 1022-1027. https://doi.org/10.1016/S0886-3350(00)00317-5

[3] Mendicute, J., Irigoyen, C., Aramberri, J., Ondarra, A. and Montés-Micó, R. (2008) Foldable Toric Intraocular Lens for Astigmatism Correction in Cataract Patients. Journal of Cataract \& Refractive Surgery, 34, 601-607. https://doi.org/10.1016/j.jcrs.2007.11.033

[4] Sun, X.Y., Vicary, D., Montgomery, P. and Griffiths, M. (2000) Toric Intraocular Lenses for Correcting Astigmatism in 130 Eyes. Ophthalmology, 107, 1776-1781 https://doi.org/10.1016/S0161-6420(00)00266-9

[5] Olsen, T. (1986) On the Calculation of Power from Curvature of the Cornea. British Journal of Ophthalmology, 70, 152-154. https://doi.org/10.1136/bjo.70.2.152

[6] Visser, N., Berendschot, T.T., Verbakel, F., de Brabander, J. and Nuijts, R.M. (2012) Comparability and Repeatability of Corneal Astigmatism Measurements Using Different Measurement Technologies. Journal of Cataract \& Refractive Surgery, 38, 1764-1770. https://doi.org/10.1016/j.jcrs.2012.05.036

[7] Ho, J.D., Tsai, C.Y. and Liou, S.W. (2009) Accuracy of Corneal Astigmatism Estimation by Neglecting the Posterior Corneal Surface Measurement. American Journal of Ophthalmology, 147, 788-795. https://doi.org/10.1016/j.ajo.2008.12.020

[8] Koch, D.D., Ali, S.F., Weikert, M.P., Shirayama, M., Jenkins, R. and Wang, L. (2012) Contribution of Posterior Corneal Astigmatism to Total Corneal Astigmatism. 
Journal of Cataract \& Refractive Surgery, 38, 2080-2087. https://doi.org/10.1016/j.jcrs.2012.08.036

[9] Hasegawa, Y., Okamoto, F., Nakano, S., Hiraoka, T. and Oshika, T. (2013) Effect of Preoperative Corneal Astigmatism Orientation on Results with a Toric Intraocular Lens. Journal of Cataract \& Refractive Surgery, 39, 1846-1851. https://doi.org/10.1016/j.jcrs.2013.06.019

[10] Ernest, P. and Potvin, R. (2011) Effects of Preoperative Corneal Astigmatism Orientation on Results with a Low-Cylinder-Power Toric Intraocular Lens. Journal of Cataract \& Refractive Surgery, 37, 727-732. https://doi.org/10.1016/j.jcrs.2010.11.026

[11] Zhang, L., Sy, M.E., Mai, H., Yu, F. and Hamilton, D.R. (2015) Effect of Posterior Corneal Astigmatism on Refractive Outcomes after Toric Intraocular Lens Implantation. Journal of Cataract \& Refractive Surgery, 41, 84-89. https://doi.org/10.1016/j.jcrs.2014.04.033

[12] Savini, G. and Naeser, K. (2015) An Analysis of the Factors Influencing the Residual Refractive Astigmatism after Cataract Surgery with Toric Intraocular Lenses. Investigative Ophthalmology \& Visual Science, 56, 827-835.

https://doi.org/10.1167/iovs.14-15903

[13] Goggin, M., Zamora-Alejo, K., Esterman, A. and van Zyl, L. (2015) Adjustment of Anterior Corneal Astigmatism Values to Incorporate the Likely Effect of Posterior Corneal Curvature for Toric Intraocular Lens Calculation. Journal of Refractive Surgery, 31, 98-102. https://doi.org/10.3928/1081597X-20150122-04

[14] Eydelman, M.B., Drum, B., Holladay, J., et al. (2006) Standardized Analyses of Correction of Astigmatism by Laser Systems that Reshape the Cornea. Journal of Refractive Surgery, 22, 81-95. https://doi.org/10.3928/1081-597X-20060101-16

[15] Park, C.Y., Chuck, R.S., Channa, P., Lim, C.Y. and Ahn, B.J. (2011) The Effect of Corneal Anterior Surface Eccentricity on Astigmatism after Cataract Surgery. Ophthalmic Surgery, Lasers and Imaging Retina, 42, 408-415. https://doi.org/10.3928/15428877-20110623-01

[16] Davison, J.A. and Potvin, R. (2015) Refractive Cylinder Outcomes after Calculating Toric Intraocular Lens Cylinder Power Using Total Corneal Refractive Power. Clinical Ophthalmology, 9, 1511-1517. https://doi.org/10.2147/OPTH.S88693

[17] Gudmundsdottir, E., Arnarsson, A. and Jonasson, F. (2005) Five-Year Refractive Changes in an Adult Population: Reykjavik Eye Study. Ophthalmology, 112, 672-677. https://doi.org/10.1016/j.ophtha.2004.11.039 